\title{
A Horn type spectral problem ${ }^{1}$
}

\author{
Stanislav POPOVYCH
}

Department of Mathematics, Chalmers University of Technology, SE-412 96 Göteborg, Sweden E-mail: stanislp@math.chalmers.se

\section{Abstract}

The famous Horn's problem is about the possible eigenvalue list of a sum of two Hermitian matrices with prescribed eigenvalue lists. The Spectral Problem is to describe possible spectra for an irreducible finite family of Hermitian operators with the sum being a scalar operator. In case when spectra consist of finite number of points the complexity of the problem depends on properties of some rooted tree. We will consider the cases for which the explicit answer on the Spectral Problem can be obtained.

2000 MSC: 16W10, 16G20, 47L30

\section{Introduction}

Let $A_{1}, A_{2}, A_{3}$ be Hermitian $n \times n$ matrices with given lists of eigenvalues

$$
\lambda\left(A_{j}\right)=\left\{\lambda_{1}\left(A_{j}\right) \geq \lambda_{2}\left(A_{j}\right) \geq \ldots \geq \lambda_{n}\left(A_{j}\right)\right\}
$$

The well-known classical problem about the spectrum of a sum of two Hermitian $n \times n$ matrices (Alfred Horn's problem) is to describe possible values of $\lambda\left(A_{1}\right), \lambda\left(A_{2}\right), \lambda\left(A_{3}\right)$ such that

$$
A_{1}+A_{2}=A_{3}
$$

This problem was solved recently by Klyachko, Totaro, Knutson and Tao [2]. We shall briefly recall the solution below.

Let $\alpha=\lambda\left(A_{1}\right), \beta=\lambda\left(A_{2}\right)$ and $\gamma=\lambda\left(A_{3}\right)$. One obvious necessary condition is the following "trace equality":

$$
\sum_{i=1}^{n} \alpha_{i}+\sum_{j=1}^{n} \beta_{j}=\sum_{k=1}^{n} \gamma_{k}
$$

It turns out that necessary and sufficient conditions can be given in terms of linear inequalities of the form:

$$
\sum_{i \in I} \alpha_{i}+\sum_{j \in J} \beta_{j} \geq \sum_{k \in K} \gamma_{k}
$$

where $I, J, K$ are certain subsets of $\{1, \ldots, n\}$ of the same cardinality $r<n$.

To describe such triples $(I, J, K)$ we recall the standard correspondence between subsets and partitions. A subset $I=\left\{i_{1}<i_{2} \ldots<i_{r}\right\} \subset\{1, \ldots, n\}$ corresponds to a partition

$$
\lambda(I)=\left\{i_{r}-r, i_{r-1}-(r-1), \ldots, i_{1}-1\right\}
$$

of length at most $r$ consisting of nonnegative integers.

Let $\alpha, \beta$ and $\gamma$ be three partitions and $c_{\alpha, \beta}^{\gamma}$ be the corresponding Littlewood-Richardson coefficient. Set

$$
R_{r}^{n}=\left\{(I, J, K) \mid c_{\lambda(I), \lambda(J)}^{\lambda(K)}=1\right\}
$$

The solution of the Horn's problem is given by

\footnotetext{
${ }^{1}$ Presented at the $3^{\text {rd }}$ Baltic-Nordic Workshop "Algebra, Geometry, and Mathematical Physics “, Göteborg, Sweden, October 11-13, 2007.
} 
Theorem $1.1([2])$. A triple $(\alpha, \beta, \gamma)$ is a triple of lists of eigenvalues of three Hermitian matrices $A_{1}, A_{2}, A_{3}$ such that

$$
A_{1}+A_{2}=A_{3}
$$

if and only if inequalities (1.1) are satisfied for all $r<n$ and $(I, J, K) \in R_{r}^{n}$ and the "trace equality"

$$
\sum_{i=1}^{n} \alpha_{i}+\sum_{i=1}^{n} \beta_{i}=\sum_{i=1}^{n} \gamma_{i}
$$

holds. In fact the resulting system of inequalities together with "trace equality" is a complete and independent set of conditions.

The Littlewood-Richardson rule provides an algorithm to compute the sets $R_{r}^{n}$ for a given $n$. In fact the explicit recursive answer to Horn's problem can be given using larger set of triples $T_{r}^{n}$ instead of $R_{r}^{n}$. That this is possible was conjectured by Horn in 1962 and proved by Klyachko, Totaro, Knutson and Tao (see overview [2]). For a triple $(I, J, K)$ of subsets of $\{1, \ldots, n\}$, Horn defined sets $T_{r}^{n}$ of triples $(I, J, K)$ of subsets of $\{1, \ldots, n\}$ of the same cardinality $r$, by the following recursive procedure. Set

$$
U_{r}^{n}=\left\{(I, J, K) \mid \sum_{i \in I} i+\sum_{j \in J} j=\sum_{k \in K} k+\frac{r(r+1)}{2}\right\}
$$

When $r=1$, set $T_{1}^{n}=U_{1}^{n}$. Otherwise, let

$$
T_{r}^{n}=\left\{(I, J, K) \in U_{r}^{n} \mid \sum_{f \in F} i_{f}+\sum_{g \in G} j_{g} \leq \sum_{h \in H} k_{h}+\frac{p(p+1)}{2}, \text { for all } p<r \text { and }(F, G, H) \in T_{p}^{r}\right\}
$$

The result of Klyatchko, Totaro, Knutson and Tao is that $(\alpha, \beta, \gamma)$ is a triple of lists of eigenvalues of three Hermitian matrices $A_{1}, A_{2}, A_{3}$ such that $A_{1}+A_{2}=A_{3}$ if and only if inequality (1.1) holds for every triple $(I, J, K) \in \bigcup_{r=1}^{n-1} T_{r}^{n}$.

\section{Spectral Problem}

A modification of the Horn's problem is the Spectral Problem posed in [5]. The Spectral Problem is to describe a connection between subsets of real numbers $M_{1}, M_{2}, \ldots, M_{n}$ and $\gamma \in \mathbb{R}$ necessary and sufficient for the existence of Hermitian operators $A_{1}, A_{2}, \ldots, A_{n}$ such that

$$
A_{1}+A_{2}+\ldots+A_{n}=\gamma I
$$

and

$$
\lambda\left(A_{1}\right)=M_{1}, \quad \lambda\left(A_{2}\right)=M_{2}, \quad \ldots, \quad \lambda\left(A_{n}\right)=M_{n}
$$

For example when $\lambda\left(A_{j}\right)=\{0,1\}$ we have the following problem. Describe $\gamma \in \mathbb{R}$ such that there orthoprojectors $P_{1}, \ldots, P_{n}$ with

$$
P_{1}+\ldots+P_{n}=\gamma I
$$

Let

$$
\Sigma_{n}=\left\{\gamma \mid \text { there are orthoprojections } P_{j} \text { such that } P_{1}+\ldots+P_{n}=\gamma I\right\}
$$


Clearly $\Sigma_{n} \subseteq[0, n]$. The following beautiful description of $\Sigma_{n}$ was obtained in [7]:

$$
\Sigma_{n}=\underbrace{\left(\left\{\phi^{+k}(1) \mid k \geq 0\right\} \cup\left\{\phi^{+k}(0) \mid k \geq 0\right\}\right)}_{X_{n}} \cup\left[\beta_{n}, n-\beta_{n}\right] \cup\left(n-X_{n}\right)
$$

Here $\beta_{n}=\left(n-\sqrt{n^{2}-4 n}\right) / 2$ is a fixed point of the dynamical system

$$
\phi^{+}(\alpha)=1+\frac{1}{n-1-\alpha}
$$

In this work the sets $M_{1}, M_{2}, \ldots, M_{n}$ will be finite. Even for finite $M_{k}$ it can be very complicated to describe such $n$-tuples of operators up to unitary equivalence if the cardinality of $M_{k}$ is large enough. More precisely the corresponding $*$-algebras defined below may be $*$-wild (see [10]).

Let us stress here that an essential difference with Horn's classical problem is that we do not fix the dimension of $H$ and the spectral multiplicities.

The Spectral Problem can be stated in terms of $*$-representations of $*$-algebras introduced in [9]. Namely, let $\alpha^{(j)}=\left(\alpha_{1}^{(j)}, \alpha_{2}^{(j)}, \ldots, \alpha_{m_{j}}^{(j)}, 0\right)(1 \leq j \leq n)$ be vectors with positive strictly decreasing coefficients. Put $M_{j}=\alpha^{(j)}$. Let us consider the associative algebra defined by the following generators and relations :

$$
\begin{aligned}
& \mathcal{A}_{M_{1}, \ldots, M_{n}, \gamma}=\mathbb{C}\left\langle p_{1}^{(1)}, p_{2}^{(1)}, \ldots, p_{m_{1}}^{(1)}, p_{1}^{(2)}, p_{2}^{(2)}, \ldots, p_{m_{2}}^{(2)}, \ldots, p_{1}^{(n)}, p_{2}^{(n)}, \ldots, p_{m_{n}}^{(n)}\right| p_{k}^{(i) 2}=p_{k}^{(i)}, \\
&\left.\sum_{i=1}^{n} \sum_{k=1}^{m_{i}} \alpha_{k}^{(i)} p_{k}^{(i)}=\gamma e, p_{j}^{(i)} p_{k}^{(i)}=0, j, k=1, \ldots, m_{i}, j \neq k, i=1, \ldots, n\right\rangle
\end{aligned}
$$

Here $e$ is the identity of the algebra. This is a $*$-algebra if we declare all generators to be self-adjoint. Equivalently this algebra can be given by the following generators and relations

$$
A_{M_{1}, \ldots, M_{n}, \gamma}=\left\langle A_{1}, \ldots, A_{n} \mid A_{1}+\ldots+A_{n}=\gamma e, P_{1}\left(A_{1}\right)=0, \ldots, P_{n}\left(A_{n}\right)=0\right\rangle
$$

where $P_{k}$ is a polynomial with simple roots from the set $M_{k}$.

We can associate a star-shaped graph $G$ with $n$ rays of lengths $m_{1}, \ldots, m_{n}$ coming from a single center. We will label the vertices of this graph by the points of the sets $M_{1}, \ldots, M_{n}$ to associated a labeled graph with the algebra $A_{M_{1}, \ldots, M_{n}, \gamma}$ which completely determines the algebra. We can write $A_{M_{1}, \ldots, M_{n}, \gamma}=A_{G, \chi}$ where $\chi$ is the labeling of the graph $G$, i.e. $\chi$ assigns real values to each vertex of the graph in such a way that $\chi$ assigns all values from $M_{k} \backslash\{0\}$ to the $k$-th ray such that they increase to the center. To the central vertex $\chi$ assigns value $\gamma$. We will fix some enumeration of the vertices of graph $G$ and thus $\chi$ will be identified with a vector with $m_{1}+\cdots+m_{n}+1$ coordinates.

The following theorem reveals a remarkable connection between complexity of the algebra $A_{G, \chi}$ and the properties of the graph $G$. Namely the complexity depends on whether $G$ is a Dynkin or a non-Dynkin graph. Recall that the Dynkin graphs are those for which $\left(m_{1}, \ldots, m_{n}\right) \in\{(2,2,2),(3,3,2),(3,4,2),(5,3,2)\}$ and the extended Dynkin graphs are those for which $\left(m_{1}, \ldots, m_{n}\right) \in\{(2,2,2,2),(3,3,3),(4,4,2),(6,3,2)\}$.

Theorem 2.1 ([11]). For a given graph $G$ the following holds:

1. If $G$ is a Dynkin graph, then algebra $A_{G, \chi}$ is finite-dimensional for all $\chi$.

2. If $G$ is extended Dynkin graph, then algebra $A_{G, \chi}$ has quadratic growth for all $\chi$.

3. If $G$ is non-Dynkin, then $A_{G, \chi}$ contains the free algebra with 2 generators (hence it has the exponential growth). 
Clearly the Spectral Problem is equivalent to a problem of a description of the set $\Sigma_{m_{1}, m_{2}, \ldots, m_{n}}$ of the parameters $\alpha_{k}^{(j)}, \gamma$ for which there exist $*$-representations of $\mathcal{A}_{M_{1}, \ldots, M_{n}, \gamma}$.

Let us call a $*$-representation $\pi$ of the algebra $\mathcal{A}_{M_{1}, \ldots, M_{n}, \gamma}$ non-degenerate if spectrum of $\pi\left(A_{k}\right)$ coincides with $M_{k}$ for all $k$.

Consider the set

$$
\Sigma_{m_{1}, \ldots, m_{n}}^{n .-d .}=\left\{\chi \mid \text { there is a non-degenerate } * \text {-representation of } \mathcal{A}_{G, \chi}\right\}
$$

which depends only on $\left(m_{1}, \ldots, m_{n}\right)$. Every irreducible representation of algebra $\mathcal{A}_{M_{1}, M_{2}, \ldots, M_{n}, \gamma}$ is an irreducible non-degenerate ${ }^{*}$-representation of an algebra $\mathcal{A}_{\widetilde{M}_{1}, \ldots, \widetilde{M}_{n}, \gamma}$ for some subsets $\widetilde{M}_{j} \subset M_{j}$. Hence $\left(M_{1}, \ldots, M_{n}, \gamma\right) \in \Sigma_{m_{1}, \ldots, m_{n}}$ if and only if there exists $\left(\widetilde{M}_{1}, \ldots, \widetilde{M}_{n}, \gamma\right) \in$ $\Sigma_{\left|\widetilde{M}_{1}\right|, \ldots,\left|\widetilde{M}_{n}\right|}^{n-d}$

Henceforth we will denote the set $\Sigma_{m_{1}, \ldots, m_{n}}$ by $\Sigma(G)$. Irreducible representations of the algebras $\mathcal{A}_{G, \chi}$ associated with the Dynkin graph $G$ exist only in certain dimensions that are bounded from above (see [8]). In [3,4] we have given a complete description of $\Sigma(G)$ for all Dynkin graphs $G$ and an algorithm for finding all irreducible representations.

\section{Coxeter functors}

The main tools for our classification are the Coxeter functors for locally-scalar graph representations. First we will recall a connection between category of *-representation of algebra $\mathcal{A}_{G, \chi}$ associated with the graph $G$ and locally-scalar representations of the graph $G$. For more details see [3].

Henceforth we will use definitions, notations and results about representations of graphs in the category of Hilbert spaces found in [8].

A graph $G$ consists of a set of vertices $G_{v}$, a set of edges $G_{e}$ and a map $\varepsilon$ from $G_{e}$ into the set of one- and two-element subsets of $G_{v}$ (the edge is mapped into the set of incident vertices). We will consider only connected finite graphs without cycles. Fix a decomposition of $G_{v}$ of the form $G_{v}=\stackrel{\circ}{G}_{v} \sqcup \stackrel{\bullet}{G}_{v}$ such that for each $\alpha \in G_{e}$ one of the vertices from $\varepsilon(\alpha)$ belongs to $\stackrel{\circ}{G}_{v}$ and the other to $\stackrel{\bullet}{G}_{v}$. Vertices in $\stackrel{\circ}{G}_{v}$ will be called even, and those in the set $\dot{G}_{v}$ odd.

Representation of $G$ associates with each vertex $g \in G_{v}$ a Hilbert space $\Pi(g)=H_{g}$, and with each edge $\gamma \in G_{e}$ such that $\varepsilon(\gamma)=\left\{g_{1}, g_{2}\right\}$ a pair of mutually adjoint operators $\Pi(\gamma)=\left\{\Gamma_{g_{1}, g_{2}}\right.$, $\left.\Gamma_{g_{2}, g_{1}}\right\}$, where $\Gamma_{g_{1}, g_{2}}: H_{g_{2}} \rightarrow H_{g_{1}}$. We now construct a category $\operatorname{Rep}(G, \mathcal{H})$. Its objects are the representations of the graph $G$ in $\mathcal{H}$. A morphism $C: \Pi \rightarrow \widetilde{\Pi}$ is a family $\left\{C_{g}\right\}_{g \in G_{v}}$ of operators $C_{g}: \Pi(g) \rightarrow \widetilde{\Pi}(g)$ such that $\Gamma_{g_{2}, g_{1}} C_{g_{2}}=C_{g_{1}} \widetilde{\Gamma}_{g_{2}, g_{1}}$.

Let $M_{g}$ be the set of vertices connected with $g$ by an edge. Define operators

$$
A_{g}=\sum_{g^{\prime} \in M_{g}} \Gamma_{g g^{\prime}} \Gamma_{g^{\prime} g}
$$

A representation $\Pi$ in $\operatorname{Rep}(G, \mathcal{H})$ will be called locally-scalar if all operators $A_{g}$ are scalar, i.e. $A_{g}=\alpha_{g} I_{H_{g}}$. The full subcategory $\operatorname{Rep}(G, \mathcal{H})$, the objects of which are locally-scalar representations, will be denoted by $\operatorname{Rep} G$ and called the category of locally-scalar representations of the graph $G$.

Denote $V_{G}=\mathbb{R}^{G_{v}}$. Elements $x$ of $V_{G}$ we will call $G$-vectors. A vector $x=\left(x_{g}\right)$ is called positive, $x>0$, if $x \neq 0$ and $x_{g} \geq 0$ for all $g \in G_{v}$. Denote $V_{G}^{+}=\left\{x \in V_{G} \mid x>0\right\}$. If $\Pi$ is a finite dimensional representation of the graph $G$ then the $G$-vector $(d(g))$, where $d(g)=\operatorname{dim} \Pi(g)$ is called the dimension of $\Pi$. If $A_{g}=f(g) I_{H_{g}}$ then the $G$-vector $f=(f(g))$ is called the character and $\Pi$ is called $f$-representation in this case. The support $G_{v}^{\Pi}$ of $\Pi$ is $\left\{g \in G_{v} \mid \Pi(g) \neq 0\right\}$. 
A character of the locally-scalar representation $\Pi$ is uniquely defined on the support $G_{v}^{\Pi}$ and non-uniquely on its complement. In the general case, denote by $\left\{f_{\Pi}\right\}$ the set of characters of $\Pi$. For each vertex $g \in G_{v}$, denote by $\sigma_{g}$ the linear operator on $V_{G}$ given by the formulae:

$$
\begin{aligned}
& \left(\sigma_{g} x\right)_{g^{\prime}}=x_{g^{\prime}}, \quad \text { if } g^{\prime} \neq g \\
& \left(\sigma_{g} x\right)_{g}=-x_{g}+\sum_{g^{\prime} \in M_{g}} x_{g^{\prime}}
\end{aligned}
$$

The mapping $\sigma_{g}$ is called the reflection at the vertex $g$. The composition of all reflections at odd vertices is denoted by $\dot{c}$ (it does not depend on the order of the factors), and at all even vertices by $\stackrel{\circ}{c}$. A Coxeter transformation is $c=\stackrel{\circ}{c} c, c^{-1}=\stackrel{\bullet}{c} \stackrel{\circ}{~ . ~ T h e ~ t r a n s f o r m a t i o n ~} \stackrel{\bullet}{c}(\stackrel{\circ}{c})$ is called an odd (even) Coxeter map. Let us adopt the following notations for compositions of the Coxeter maps: $\dot{\bullet}_{k}=\ldots \stackrel{\bullet \bullet c c}{c}(k$ factors $), \stackrel{\circ}{c} c_{k}=\ldots \stackrel{\bullet \bullet c c}{c}$ ( $k$ factors $), k \in \mathbb{N}$.

If $d(g)$ is the dimension of a locally-scalar graph representation $\Pi$, then

$$
\begin{aligned}
& \stackrel{\circ}{c}(d)(g)= \begin{cases}-d(g)+\sum_{g^{\prime} \in M_{g}} d\left(g^{\prime}\right), & \text { if } g \in \stackrel{\circ}{G}_{v} \\
d(g), & \text { if } g \in \dot{\leftrightarrow}_{v}\end{cases} \\
& \stackrel{\bullet}{c}(d)(g)= \begin{cases}-d(g)+\sum_{g^{\prime} \in M_{g}} d\left(g^{\prime}\right), & \text { if } g \in \dot{\bullet}_{v} \\
d(g), & \text { if } g \in \stackrel{\circ}{G}_{v}\end{cases}
\end{aligned}
$$

For $d \in Z_{G}^{+}$and $f \in V_{G}^{+}$, consider the full subcategory $\operatorname{Rep}(G, d, f)$ in $\operatorname{Rep} G$ (here $Z_{G}^{+}$is the set of positive integer $G$-vectors), with the set of objects $O b \operatorname{Rep}(G, d, f)=\{\Pi \mid \operatorname{dim} \Pi(g)=$ $\left.d(g), f \in\left\{f_{\Pi}\right\}\right\}$. All representations $\Pi$ from $\operatorname{Rep}(G, d, f)$ have the same support $X=X_{d}=$ $G_{v}^{\Pi}=\left\{g \in G_{v} \mid d(g) \neq 0\right\}$. Let $\stackrel{\circ}{X}=X \cap \stackrel{\circ}{G_{v}}, \stackrel{\circ}{X}=X \cap \dot{\bullet}_{v} . \operatorname{Rep}_{\circ}(G, d, f) \subset \operatorname{Rep}(G, d, f)$ $\left(\operatorname{Rep}_{\bullet}(G, d, f) \subset \operatorname{Rep}(G, d, f)\right)$ is the full subcategory with objects $(\Pi, f)$ where $f(g)>0$ if $g \in \stackrel{\circ}{X}(f(g)>0$ if $g \in \stackrel{\circ}{X})$.

Put

$$
\begin{aligned}
& \dot{c}_{d}(f)(g)=\stackrel{\circ}{f}_{d}(g)= \begin{cases}\dot{c}(f)(g), & \text { if } g \in \dot{X}_{d} \\
f(g), & \text { if } g \notin \dot{X}_{d}\end{cases} \\
& {\stackrel{\circ}{c_{d}}}_{d}(f)(g)=\stackrel{\circ}{f}_{d}(g)= \begin{cases}\stackrel{\circ}{c}(f)(g), & \text { if } g \in \stackrel{\circ}{X}_{d} \\
f(g), & \text { if } g \notin \stackrel{\circ}{X}_{d}\end{cases}
\end{aligned}
$$

Let us denote

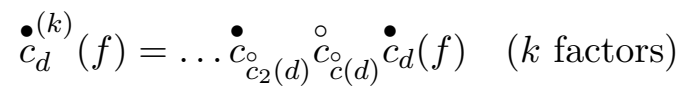

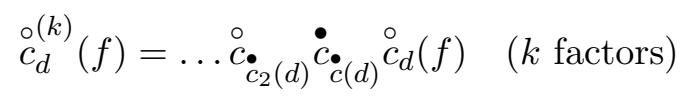

The even and odd Coxeter reflection functors are defined in [8],

$$
\begin{array}{ll}
\stackrel{\circ}{F}: \operatorname{Rep}_{\diamond}(G, d, f) \rightarrow \operatorname{Rep}_{\diamond}\left(G, \stackrel{\circ}{c}(d), \stackrel{\circ}{f_{d}}\right) & \text { if }(d, f) \in S_{\circ} \\
\stackrel{\bullet}{F}: \operatorname{Rep}_{\bullet}(G, d, f) \rightarrow \operatorname{Rep}_{\bullet}\left(G, \stackrel{\bullet}{c}(d), \stackrel{\bullet}{f}_{d}\right) & \text { if }(d, f) \in S \bullet
\end{array}
$$

These functors are equivalences of the categories. Let us denote $\stackrel{\circ}{F}_{k}(\Pi)=\ldots \stackrel{\circ}{F} \dot{\bullet} \stackrel{\circ}{F}(\Pi)(k$ factors), $\dot{F}_{k}(\Pi)=\ldots \dot{F} \stackrel{\circ}{F} \dot{F}(\Pi)$ ( $k$ factors), if the compositions exist. Using these functors, an 
analog of Gabriel's theorem for graphs and their locally-scalar representations has been proven in [8]. In particular, it has been proved that any locally-scalar graph representation decomposes into a direct sum (finite or infinite) of finite dimensional indecomposable representations, and all indecomposable representations can be obtained by odd and even Coxeter reflection functors starting from the simplest representations $\Pi_{g}$ of the graph $G\left(\Pi_{g}(g)=\mathbb{C}, \Pi_{g}\left(g^{\prime}\right)=0\right.$ if $g \neq$ $\left.g^{\prime} ; g, g^{\prime} \in G_{v}\right)$.

Here we will describe the connection of representations of the algebra $\mathcal{A}_{G, \chi}$ and locally-scalar graph representations.

Let $\pi$ be a *-representation of the algebra $\mathcal{A}_{G, \chi}$ in space $H$ of dimension $n$. Let $P_{j}^{(s)}$ denote projection $\pi\left(p_{j}^{(s)}\right)$. Let us define projections $R_{j}^{(s)}=P_{1}^{(s)}+\ldots+P_{j}^{(s)}$, and subspaces $H_{j}^{(s)}=$ $\Im \mathrm{m} R_{j}^{(s)}$. Let $\Gamma_{j}^{(s)}: H_{j}^{(s)} \rightarrow H$ be natural isometries. Then, in particular, $\Gamma_{j}^{(s) *} \Gamma_{j}^{(s)}=I_{H_{j}^{(s)}}$, $\Gamma_{j}^{(s)} \Gamma_{j}^{(s) *}=R_{j}^{(s)}$. Let $V_{j}^{(s)}$ denote the operator $\Gamma_{j+1}^{(s) *}\left(\sum_{i=1}^{j} \xi_{s, i}^{(j)} P_{i}^{(s)}\right) \Gamma_{j}^{(s)}$ acting from $H_{j}^{(s)}$ to $H_{j+1}^{(s)}$, where $1 \leq j \leq m_{s}-1$ and coefficients $\xi_{s, i}^{(j)}$ for a fixed $S$ are defined by the following recursion: $\xi_{s, k}^{(l-1)}=\sqrt{\xi_{s, l}^{(l) 2}-\xi_{s, k}^{(l) 2}}$, with initial data $\xi_{s, m_{s}}^{\left(m_{s}\right)}=\lambda_{1}^{(s)}, \xi_{s, m_{s}-1}^{\left(m_{s}\right)}=\lambda_{2}^{(s)}, \ldots, \xi_{s, 1}^{\left(m_{s}\right)}=\lambda_{m_{s}}^{(s)}$. It is easy to check that if $\lambda_{1}^{(s)} \geq \ldots \lambda_{m_{s}}^{(s)} \geq 0$ then the above recursion determines $\xi_{s, i}^{(j)}$ uniquely. Then $V_{j}^{(s) *} V_{j}^{(s)}+V_{j-1}^{(s)} V_{j-1}^{(s) *}=\xi_{s, j}^{(j) 2} I_{H_{j}^{(s)}}, V_{1}^{(s) *} V_{1}^{(s)}=\xi_{s, 1}^{(1) 2}, V_{m_{s}}^{(s)} V_{m_{s}}^{(s) *}=A^{(s)}$. Here $A^{(s)}: H \rightarrow H$ is a self-adjoint operator. Moreover, $A^{(1)}+\ldots+A^{(t)}=\gamma I_{H}$. Operators $V_{j}^{(s)}$ together with their conjugate give raise to a locally-scalar representation of the graph $G$ with a character with coefficients of $\xi_{s, i}^{(j) 2}$ and $\gamma$ appropriately ordered.

This correspondence is in fact an equivalence functor between category of non-degenerate *representations of algebra $\mathcal{A}_{G, \chi}$ and the category of non-degenerate locally-scalar representations of the graph $G$.

Since we will be concerned with extended Dynkin star-shaped graphs we will simplify simplify notations and consider only graphs with three rays. This will exclude graph $\widetilde{D}_{4}$ for which the formulae are analogues and are left to be recovered by the reader.

So we will use notations $\alpha, \beta, \delta$ instead of $\alpha^{(1)}, \alpha^{(2)}, \alpha^{(3)}$. By $\chi$ we will denote the vector $\left(\alpha_{1}, \alpha_{2}, \ldots, \alpha_{k}, \beta_{1}, \beta_{2}, \ldots, \beta_{l}, \delta_{1}, \delta_{2}, \ldots, \delta_{m}, \gamma\right)$.

Definition 3.1. A finite-dimensional $*$-representation $\pi$ of the algebra $\mathcal{A}_{G, \chi}$ such that $\pi\left(p_{i}\right) \neq 0$ for $1 \leq i \leq k, \pi\left(q_{j}\right) \neq 0$ for $1 \leq j \leq l, \pi\left(s_{d}\right) \neq 0$ for $1 \leq d \leq m$ and $\sum_{i=1}^{k} \pi\left(p_{i}\right) \neq I$, $\sum_{j=1}^{l} \pi\left(q_{j}\right) \neq I, \sum_{d=1}^{m} \pi\left(s_{d}\right) \neq I$ will be called non-degenerate. By $\overline{\operatorname{Rep}} \mathcal{A}_{G, \chi}$ we will denote the full subcategory of non-degenerate representations in the category $\operatorname{Rep} \mathcal{A}_{G, \chi}$ of $*$-representations of the $*$-algebra $\mathcal{A}_{G, \chi}$.

The above functor transform the representation of the algebra with character $(\alpha, \beta, \delta, \gamma)$ to locally-scalar representation of the graph $G$ with the following character $f: f\left(g_{0}\right)=\gamma$ and on the first ray $f\left(g_{k}\right)=\alpha_{1}, f\left(g_{k-1}\right)=\alpha_{1}-\alpha_{k}, f\left(g_{k-2}\right)=\alpha_{2}-\alpha_{k}, f\left(g_{k-3}\right)=\alpha_{2}-\alpha_{k-1}, f\left(g_{k-4}\right)=$ $\alpha_{3}-\alpha_{k-1}, \ldots$ It is clear by analogy how to define $f$ on other two rays.

A locally-scalar representation of the graph $G$ with the character $f\left(g_{i}\right)=x_{i} \in \mathbb{R}^{*}$ corresponds to a non-degenerate representation of $\mathcal{A}_{G, \chi}$ with the character

$$
\begin{aligned}
\alpha_{1} & =x_{k} \\
\alpha_{k} & =x_{k}-x_{k-1} \\
\alpha_{2} & =x_{k}-x_{k-1}+x_{k-2} \\
\alpha_{k-1} & =x_{k}-x_{k-1}+x_{k-2}-x_{k-3} \\
\alpha_{3} & =x_{k}-x_{k-1}+x_{k-2}-x_{k-3}+x_{k-4}
\end{aligned}
$$


Here $x_{j}=0$ if $j \leq 0$. Analogously one can find $\beta_{j}$ and $\delta_{t}$. We will denote $\Pi$ by $\Phi(\pi)$.

The mentioned above functor $\Phi$ acts between categories $\overline{\operatorname{Rep}} \mathcal{A}_{G, \chi}$ and $\operatorname{Rep} G$, see [4].

The representation $\Pi$ is unitary equivalent to an irreducible representation from the image of the functor $\Phi$ if and only if

$$
\begin{aligned}
& 0<x_{1}<x_{2}<\ldots<x_{k}, \quad 0<x_{k+1}<x_{k+2}<\ldots<x_{k+l} \\
& 0<x_{k+l+1}<x_{k+l+2}<\ldots<x_{k+l+m} \\
& 0<d_{1}<d_{2}<\ldots<d_{k}<d_{0}, \quad 0<d_{k+1}<d_{k+2}<\ldots<d_{k+l}<d_{0} \\
& 0<d_{k+l+1}<d_{k+l+2}<\ldots<d_{k+l+m}<d_{0}
\end{aligned}
$$

The vector $n=\left(n_{0}, n_{1}, \ldots, n_{k+l+m}\right)$ is called the generalized dimension of the representation $\pi$ of the algebra $\mathcal{A}_{G, \chi}$. Let $\Pi=\Phi(\pi)$ for a non-degenerate representation of the algebra $\mathcal{A}_{G, \chi}$, $d=\left(d_{1}, \ldots, d_{k+l+m}, d_{0}\right)$ be the dimension of $\Pi$. It is easy to see that

$$
\begin{aligned}
n_{1}+n_{2}+\ldots+n_{k} & =d_{k} \\
n_{2}+\ldots+n_{k-1}+n_{k} & =d_{k-1} \\
n_{2}+\ldots+n_{k-1} & =d_{k-2} \\
n_{3}+\ldots+n_{k-2}+n_{k-1} & =d_{k-3}
\end{aligned}
$$

\section{Spectral problem for algebras associated with extended Dynkin graphs}

Let us recall a few facts about root systems associated with extended Dynkin diagrams. Let $G$ be a simple connected graph. Then its Tits form is

$$
q(\alpha)=\sum_{i \in G_{v}} \alpha_{i}^{2}-\frac{1}{2} \sum_{\beta \in G_{e},\{i, j\}=\epsilon(\beta)} \alpha_{i} \alpha_{j}, \quad \alpha \in V_{G}
$$

The symmetric bilinear form is $(\alpha, \beta)=q(\alpha+\beta)-q(\alpha)-q(\beta)$. The vector $\alpha \in V_{G}$ is called sincere if each component is non-zero.

It is well known that for Dynkin graphs (and only for them) bilinear form $(\cdot, \cdot)$ is positive definite. The form is positive semi-definite for extended Dynkin graphs. And in the letter case $\operatorname{Rad} q=\{v \mid q(v)=0\}$ is equal to $\mathbb{Z} \delta$ where $\delta$ is a minimal imaginary root. For other graphs (which are neither Dynkin nor extended Dynkin) there are vectors $\alpha \geq 0$ such that $q(\alpha)<0$ and $\left(\alpha, \epsilon_{j}\right) \leq 0$ for all $j$.

For an extended Dynkin graph $G$ a vertex $j$ is called extending if $\delta_{j}=1$. The graph obtained by deleting extending vertex is the corresponding Dynkin graph. The set of roots is $\Delta=\left\{\alpha \in V_{G} \mid \alpha_{i} \in \mathbb{Z}\right.$ for all $\left.i \in G_{v}, \alpha \neq 0, q(\alpha) \leq 0\right\}$. A root $\alpha$ is real if $q(\alpha)=1$ and imaginary if $q(\alpha)=0$. Every root is either positive or negative, i.e. all coordinates are simultaneously non-negative or non-positive.

It is known that for an extended Dynkin graph the set $\Delta \cup\{0\} / \mathbb{Z} \delta$ is finite. Moreover, if $e$ is an extending vertex then the set $\Delta_{f}=\left\{\alpha \in \Delta \cup\{0\} \mid \alpha_{e}=0\right\}$ is a complete set of representatives of the cosets from $\Delta \cup\{0\} / \mathbb{Z} \delta$. If $\alpha$ is a root then $\alpha+\delta$ is again a root. We call a coset $\alpha+\delta \mathbb{Z}$ the $\delta$-series and a coset $\alpha+2 \delta \mathbb{Z}$ the $2 \delta$-series. If $\alpha$ is a root then its images under the action of the group generated by $\stackrel{\circ}{c}$ and $\dot{c}$ will be called a Coxeter series or $C$-series for short. It turns out that each $C$-series decomposes into a finite number of $\delta$-series or $2 \delta$-series of roots.

Note that to find formulae of the locally-scalar representations of a given extended Dynkin graph we need to consider two principally different cases: the case when the vector of generalized 
dimension is a real root and the case when it is an imaginary root. In the letter case the vector of parameters $\chi$ must be orthogonal to a imaginary root. Hence $\chi$ must belong to a ceratin hyperplane $h_{G}$ which depends only on the graph $G$.

It is know (see [11]) that in case $\chi \in h_{G}$ the dimension of any irreducible representation is bounded (by 2 for $\widetilde{D}_{4}$, by 3 for $\widetilde{E}_{6}$, by 4 for $\widetilde{E}_{7}$ and by 6 for $\widetilde{E}_{8}$ ). Thus in case $\chi \in h_{G}$ we can describe the set of admissible parameters $\chi$ using Horn's inequalities. In case $\chi \notin h_{g}$ the dimension of any irreducible locally-scalar representation is a real root. In what follows we will relay on the following result [6].

Theorem 4.1. Let $\pi$ be an irreducible non-degenerate *-representation of the algebra $\mathcal{A}_{G, \chi, \lambda}$ associated with an extended Dynkin graph $G$ and $\widehat{\pi}$ be the corresponding locally-scalar representation of the graph $G$. Then either generalized dimension $d$ of $\widehat{\pi}$ is a singular root or vectorparameter $(\chi, \lambda) \in h_{G}$.

For a vector $v=\left(v_{0}, \ldots, v_{n}\right)$ and $0 \leq s \leq n$ we shall write $v \geq_{s} 0$ if $v_{j}>0$ for all $j \neq s$ and $v_{s}=0$.

The equivalence functor $\Phi$ assigns to every representation $\pi \in \mathcal{A}_{G, \chi}$ of generalized dimension $\left(l_{1}, \ldots, l_{n}\right)$ a unique locally-scalar representation of graph $G$ with a character $\left(x_{1}, \ldots, x_{n}, x_{0}\right)$ and dimension $\left(v_{1}, \ldots, v_{n}, v_{0}\right)$. Let $M_{f}$ denote the transition matrix which transform the vector $\chi$ to $\left(x_{1}, \ldots, x_{n}, x_{0}\right)$, i.e. $M_{f} \chi^{t}=\left(x_{1}, \ldots, x_{n}, x_{0}\right)^{t}$ (where $v^{t}$ denote the transposed vector $v$ ). Let $M_{d}$ be the transition matrix which transforms generalized dimension $\left(v_{1}, \ldots, v_{n}, v_{0}\right)$ of a graph representation to generalized dimension $\left(l_{1}, \ldots, l_{n}\right)$ of the corresponding algebra representation, i.e. $M_{d}\left(v_{1}, \ldots, v_{n}, v_{0}\right)^{t}=\left(l_{1}, \ldots, l_{n}\right)^{t}$. Further we will omit $t$ superscript and $M_{f} v$ instead of $M_{f} v^{t}$.

Theorem 4.2. Let $G$ be an extended Dynkin graph and $\pi$ be a non-degenerate irreducible *representation of a generalized dimension $v$ of $\mathcal{A}_{G, \chi}$ for some character $\chi$. Then one of the two possibilities holds:

- $\chi \in h_{G}$ and $d=M_{d} \delta$ where $\delta$ is the minimal imaginary root of the root system associated with $G$.

- There exist $k$ and $t$ such that

$$
\stackrel{\bullet}{c}_{d}^{(k)} M_{f} \chi \geq_{t} 0, \quad \dot{c}_{k}\left(M_{d}^{-1} v\right)=e_{t}
$$

or

$$
\stackrel{\circ}{c}_{d}^{(k)} M_{f} \chi \geq_{t} 0, \quad \stackrel{\circ}{c}_{k}\left(M_{d}^{-1} v\right)=e_{t}
$$

(depending on the parity of $k+t$ ). Moreover, systems of inequalities (4.1), (4.2) are necessary and sufficient conditions for existence of representation of $\mathcal{A}_{G, \chi}$ in dimension $v$.

The explicit answers to the Spectral Problem for all extended Dynkin graphs will appear in $[6]$.

\section{References}

[1] W. Crawley-Boevey and Ch. Geiss. Horn's problem and semi-stability for quiver representations. In "Representations of Algebras. Vol. I, II. Proc. 9th Int. Con. Beijing, August 21-September 1, 2000". T. Happel, V. B. Zhong, Eds., Beijing Norm. Univ. Press, Beijing, 2002, 40-48.

[2] W. Fulton. Eigenvalues, invariant factors, highest weights and Schubert calculus. Bull. Amer. Math. Soc. 37 (2000), 209-249. 
[3] S. A. Kruglyak, S. V. Popovych, and Yu. S. Samoǐlenko. The spectral problem and *-representations of algebras associated with Dynkin graphs. J. Algebra Appl. 4 (2005), 761-776.

[4] S. A. Kruglyak, S. V. Popovych, and Yu. S. Samoĭlenko. The spectral problem and algebras associated with extended Dynkin graphs. I. Methods Funct. Anal. Topology, 11 (2005), 383-396.

[5] S. A. Kruglyak, S. V. Popovych, and Yu. S. Samoĭlenko. Representations of $*$-algebras associated with Dynkin diagrams. Uchenye Zapiski Tavricheskogo Natsional'nogo Universiteta, 16 (2003), 132139.

[6] S. V. Popovych, The Spectral Problem and algebras associated with extended Dynkin graphs, to be published.

[7] S. A. Kruglyak, V. I. Rabanovich, and Yu. S. Samoĩlenko. On sums of projections. Funct. Anal. Appl. 36 (2002), 182-195.

[8] S. A. Kruglyak and A. V. Roiter. Locally scalar representations of graphs in the category of Hilbert spaces. Funct. Anal. and Appl. 39 (2005), 91-105.

[9] S. V. Popovych, Yu. S. Samoilenko. On homomorphisms of algberas generated by projectors and Coxeter functors. Ukr. Math. Journ. 55 (2003), 1224-1237.

[10] S. Popovych, L. Turowska. Unitarizable and non-unitarizable representations of algebras generated by idempotents. J. Algebra and Discrete Math., (2004), 111-125.

[11] M. Vlasenko, A. Mellit, and Yu. Samoǐlenko. On algebras generated by linearly dependent generators that have given spectra. Funct. Anal. and Appl. 39 (2005), 14-27

Received December 20, 2007

Revised March 19, 2008 